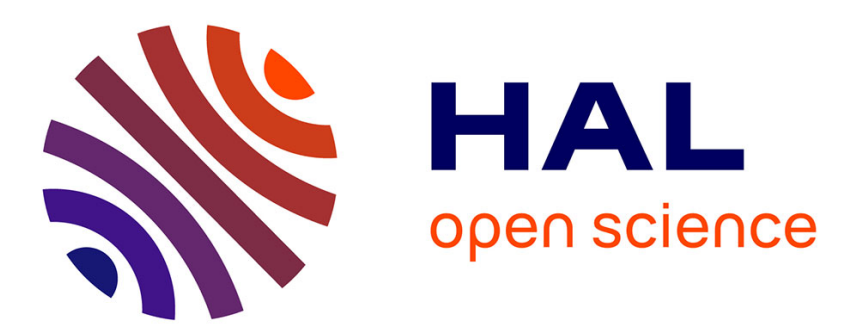

\title{
Impact of the motion and visual complexity of the background on players' performance in video game-like displays
}

\author{
Loïc Caroux, Ludovic Le Bigot, Nicolas Vibert
}

\section{To cite this version:}

Loïc Caroux, Ludovic Le Bigot, Nicolas Vibert. Impact of the motion and visual complexity of the background on players' performance in video game-like displays. Ergonomics, 2013, 56, pp.1863 1876. 10.1080/00140139.2013.847214 . hal-01470744

HAL Id: hal-01470744

https://hal.science/hal-01470744

Submitted on 17 Feb 2017

HAL is a multi-disciplinary open access archive for the deposit and dissemination of scientific research documents, whether they are published or not. The documents may come from teaching and research institutions in France or abroad, or from public or private research centers.
L'archive ouverte pluridisciplinaire HAL, est destinée au dépôt et à la diffusion de documents scientifiques de niveau recherche, publiés ou non, émanant des établissements d'enseignement et de recherche français ou étrangers, des laboratoires publics ou privés. 


\section{PRE PRINT}

Caroux, L., Le Bigot, L., \& Vibert, N. (2013). Impact of the motion and visual complexity of the background on players' performance in video game-like displays. Ergonomics, 56, 18631876. doi:10.1080/00140139.2013.847214

This is an Author's Original Manuscript of an article whose final and definitive form, the Version of Record, has been published in ERGONOMICS [copyright Taylor \& Francis], available online at: http://www.tandfonline.com/10.1080/00140139.2013.847214

\section{Impact of the motion and visual complexity of the background on players' performance in video game-like displays}

Authors:

Loïc CAROUX, Ludovic LE BIGOT, Nicolas VIBERT

Affiliation:

Centre de Recherches sur la Cognition et l'Apprentissage (CeRCA)

UMR 7295 - University of Poitiers / University of Tours / CNRS

France

Loïc Caroux is currently affiliated to the Game Innovation Lab, Polytechnic Institute of New

York University, USA

Corresponding author:

Loïc Caroux,

Game Innovation Lab, NYU-Poly

5 Metrotech Center, Room 102

Brooklyn, NY 11201

USA

Email: lcaroux@poly.edu 


\begin{abstract}
The visual interfaces of virtual environments such as video games often show scenes where objects are superimposed on a moving background. Three experiments were designed to better understand the impact of the complexity and/or overall motion of two types of visual backgrounds often used in video games on the detection and use of superimposed, stationary items. The impact of background complexity and motion was assessed during two typical video game tasks: a relatively complex visual search task and a classic, less demanding shooting task. Background motion impaired participants' performance only when they performed the shooting game task, and only when the simplest of the two backgrounds was used. In contrast, and independently of background motion, performance on both tasks was impaired when the complexity of the background increased. Eye movement recordings demonstrated that most of the findings reflected the impact of low-level features of the two backgrounds on gaze control.
\end{abstract}

\title{
Keywords
}

Shooting task, visual search, low-level visual features, eye movements, virtual environments

\section{Practitioner Summary}

Variations in the low-level features of visual backgrounds may be used to control challenge in video games. Using a visually complex background will always increase game difficulty. For the simplest game tasks, background motion will also impair users' performance if background complexity is low. 


\section{Introduction}

The use of virtual environments such as video games involves complex user-system interactions that are mainly mediated through the visual, auditory and haptic modalities (Stanney et al. 2003). However, few studies have addressed the question of how users interact with the complex, dynamic visual displays that characterize video games (Stanney, Mourant, and Kennedy 1998). A better understanding of users' interactions with dynamic visual displays should help designers to optimize the visual interfaces of virtual environments and enhance the quality of user-system interaction (Bae et al. 2012; Caroux, Le Bigot, and Vibert 2011; Dyson 2010; Hou et al. 2012; Jie and Clark 2008; Pölönen, Järvenpää, and Bilcu 2013; Sabri et al. 2007; Wolfson and Case 2000; Yannakakis, Martínez, and Jhala 2010). Most video game displays include a main action zone where the player sees objects with which she or he must interact (e.g., avatars, enemies, targets), a scene background (e.g., building interiors, landscapes), and superimposed contextual information (e.g., score or status bar). All the elements in the display may change, appear or disappear at any time. In particular, the overall scene background can move either periodically or continuously. Video games players perceive motion information through a virtual camera (Yannakakis, Martínez, and Jhala 2010), which may be controlled by the player or may move automatically to reflect the displacements of the player's avatar. Whatever the case, all camera movements involve an overall motion of the visual scene and scene background. In virtual environments, and especially in video games, two kinds of background motion are often used: Forward or backward motion is simulated by a radial expansion or contraction of the background, whereas lateral motion is simulated by a lateral motion of the overall background in the opposite direction $^{1}$ (Bubka and Bonato 2010; Hutchinson and Stocks 2013; Lepecq et al.

\footnotetext{
${ }^{1}$ Examples of recent, best-selling video games that use radial background motion: racing games (e.g., F1 2012, Codemasters, 2012), or first-person shooter games (e.g., Call of Duty: Black Ops 2,
} 
2006; Trutoiu et al. 2009). The main goal of the present work was to document the impact of these two types of background motion on players' performance.

In laterally moving scenes, all the elements of the background appear on one side of the screen and then move across it to disappear on the other side. However their relative positions are not modified. Radial background motion is more complex because the elements of the background appear (or disappear) at the centre of the screen and move towards (or come from) one of the four sides. This kind of motion implies the existence of four fixed, salient lines that converge on the screen centre and separate the elements of the background according to the side of the screen to which they move (or from which they come). Hence, radially moving backgrounds are visually more complex than laterally moving ones, and the respective impacts of lateral and radial background motion on players' behaviour may therefore depend on both background motion and complexity. As detailed below, several studies have reported effects of background motion or visual complexity on performance and/or gaze behaviour in various visual tasks. However, to the best of our knowledge, the interaction between background complexity and the presence or absence of background motion has not been studied. In the present experiments, simplified video game-like displays using lateral and radial backgrounds were manipulated to study the influence of background motion and visual complexity on participants' performance. In the first two experiments, participants had to perform a relatively complex visual search task, whereas in the third, a classic target shooting task, which was simpler in terms of attentional demands, was used. Visual search tasks (Castel, Pratt, and Drummond 2005; HubertWallander et al. 2011; Wu and Spence 2013) and shooting tasks (Jie and Clark 2008) are both

Activision, 2012). Examples of recent, best-selling video games that use lateral background motion: platform games (e.g., New Super Mario Bros U, Nintendo, 2012) or shoot'em ups (e.g., Sine Mora, Microsoft Studios, 2012). 
tasks that players have to perform in many video games. Participants' eye movements were recorded to obtain further information about the way they performed these two tasks and the underlying visual processes.

\section{Impact of background motion on visual tasks}

One of the best known effects of background motion is the sensation of vection (Riecke et al. 2006; Trutoiu et al. 2009). Vection is an illusory perception of self-motion experienced by a stationary observer exposed to large-scale motion of the visual surroundings (Lepecq et al. 2006). Vection occurs when the background motion corresponds to what is really observed during self-motion, namely when the optic flow on the retina reproduces the flow induced by self-motion.

Both in natural scenes and virtual environments, large-scale motion of the visual surroundings triggers optokinetic nystagmus (OKN), which stabilizes visual input on the retina (Kim and Palmisano 2010; Riecke et al. 2006; Vansteenkiste et al. 2013). OKN is a reflexive, conjugate movement of both eyes in which two phases alternate. During the slow phase, the eyes move in the direction of the background motion, ideally at the same velocity, in order to compensate for the motion of the visual background. In the fast phase, the eyes regularly return in the opposite direction (Ilg 1997; Waespe and Schwarz 1987). During the OKN, the average gaze line is generally shifted towards the origin of the moving scene (Ilg 1997). Human observers are able to voluntarily suppress $\mathrm{OKN}$ by fixating any stationary visual item that might be superimposed on the moving visual scene (e.g., Ilg 1997; Pola, Wyatt, and Lustgarten 1992; Rubinstein and Abel 2011; Wyatt and Pola 1984).

OKN often has negative effects on observers' performance in perceptual tasks. Kaminiarz, Krekelberg, and Bremmer (2007) and Tozzi, Morrone, and Burr (2007) studied the impact of OKN in a target localization task. In both studies, participants had to localize a target that was flashed up briefly on a laterally moving, patterned background that triggered OKN. Large 
errors of target localization were observed in comparison with the fixed background.

Harrison, Thompson, and Sanderson (2010) demonstrated that background motion decreased performance in a task involving the integration of brief auditory and visual signals. The visual stimuli were shown on a head-mounted display, and the background was the real world. When the participants walked about while performing the task, their performance was worse than when they were stationary. Consequently, moving backgrounds may decrease performance in tasks that involve rapid detection of superimposed items.

However, moving visual backgrounds do not always have negative effects on performance. Menozzi and Koga (2004) studied how people read a text displayed on a laterally moving background. Background motion had no significant effect on reading times or on the eye movements performed while reading. The main difference compared with previous studies was the nature of the task performed by the participants. Reading demands a far higher level of attentional resources, and involves sustained attention to a dense array of fixed, superimposed visual objects. The fact that readers must fixate the stationary words composing the text in order to read it was probably sufficient to cancel out $\mathrm{OKN}$ and eliminate its negative impact. Likewise, Seno, Ito, and Sunaga (2011) showed that augmenting observers' attentional load by increasing the complexity of a multiple object tracking task performed on a moving background decreased the strength of self-motion perception.

Finally, observers exposed to moving visual scenes may exhibit motion sickness in addition of vection and OKN (Ji, So, and Cheung 2009). Motion sickness, which occurs when the visual system and the vestibular system that detects head movements generate conflicting sensory input, can be triggered by exposure to virtual environments such as video games (Chang et al. 2012; Chen et al. 2012; Merhi et al. 2007; Pölönen, Järvenpää, and Bilcu 2013; Stoffregen et al. 2008). In that situation, motion sickness is also named cybersickness (Bos et al. in press; Frey et al. 2007; So, Ho, and Lo 2001). Several studies have shown that 
cybersickness can impair performance in various tasks such as driving tasks (Muth, Walker, and Fiorello 2006) or visual search tasks (Golding and Kerguelen 1992).

\section{Impact of background complexity on visual tasks}

The complexity of a visual scene depends on its low-level visual features and can be evaluated using entropy, which measures the amount of information contained in an image (Asher et al. 2013; Jie and Clark 2008; Raj et al. 2005). A visual scene has high entropy when it includes many salient colours, contrasts or luminances per unit area. In contrast, low entropy visual scenes contain only a low level of distinct visual elements.

Wolfe et al. (2002) studied the performance of participants searching for particular items in visual environments of varying complexities. In such paradigms, participants have to indicate as quickly as possible if a particular item is present or absent among a set of distractors (e.g., Eckstein 2011 for a review; Huestegge and Radach 2012; Thornton and Gilden 2007; Wolfe 1998). They found that observers took longer to detect the target in complex visual environments because more time was needed to process each of the candidate targets. The time needed to process a visual item can be evaluated by analysing observers' eye movements (for a review, see Rayner 2009). Hence, the durations of eye fixations are often used to quantify the amount of attentional resources required to process items in virtual

environments. In accordance with Wolfe et al.'s (2002) findings, Henderson, Chanceaux, and Smith (2009) demonstrated that when people look at a real world scene, the mean duration of fixations increases with scene complexity. This would reflect the observer's difficulty in extracting useful visual information from a highly cluttered visual background. Hence, the mean duration of eye fixations may be a good index of the visual complexity of a display. In video games, players not only have to detect visual items, but must also interact with them. Jie and Clark (2008) manipulated the complexity of various backgrounds to evaluate its impact on performance during a shooting game task. Participants had to shoot at targets that 
appeared on a stationary, complex picture as rapidly as possible. In line with visual search data, the time needed to shoot at targets was lower when the target appeared at locations of lower visual complexity.

\section{The current study}

As stated above, the aim of the first experiments (1a and $1 \mathrm{~b})$ was to evaluate the impact of the radial and/or lateral motion of patterned backgrounds on observers' response times and eye movements during a visual search task. A laterally patterned background and a radially patterned background were built using the same, simple visual pattern, which contained as little visual information as possible. The two backgrounds were designed to trigger OKN and sensations of vection and, when moving, generated the illusion of rightward or forward movement, respectively. For the sake of convenience, they will be referred to as the "lateral" and "radial" backgrounds below. Experiment 1a compared participants" response times and eye movements obtained with the moving versions of the lateral and radial backgrounds with those obtained with the stationary version of the lateral background. Because the results were ambiguous (see below), Experiment $1 \mathrm{~b}$ was designed to directly compare the impact of the stationary and moving versions of the radial background on visual searches.

Since, according to the literature, the impact of background motion depends on the attentional load of the task performed by observers, Experiment 2 was designed to evaluate the impact of the same backgrounds on eye movements and performance in a less attention-demanding shooting task. 


\section{Experiment 1a}

According to the literature, background motion impairs visual perception only during simple tasks such as the localization of briefly flashed dots (Kaminiarz, Krekelberg, and Bremmer 2007; Tozzi, Morrone, and Burr 2007), but not during a reading task demanding more focused attention (Menozzi and Koga 2004). Because we expected the attention required for a visual search to be closer to that required to localize a single dot than that required to read a text, the main hypothesis of Experiment 1a was that search performance is lower when the background is moving than when it is stationary because of the OKN triggered by the background motion. The presence of OKN was assessed indirectly by measuring the observer's average gaze position on the screen during background motion (Caroux, Le Bigot, and Vibert 2010). As stated above indeed, the average gaze line should be shifted towards the origin of background motion when OKN is triggered (Ilg 1997). A second hypothesis was therefore that in the absence of any superimposed item, participants' average gaze orientation is deviated towards the right side of the laterally moving background and towards the centre of the radially moving background compared to the stationary background.

\section{Methods}

\section{Participants}

Twenty-eight volunteers (23 women) aged $M=19.4$ years $(S D=1.1)$ took part in the experiment. All participants had normal or corrected-to-normal vision. Eleven participants were regular video game players (i.e. had played at least once a week during the last 12 months), four were former regular players (who had played less than once a week in the last 12 months but at least once a week before), and 13 were occasional players (who had played less than once a week throughout their lives). 


\section{Apparatus}

A head-free Tobii T120 eye-tracker with a 17-inch screen (1280 x 1024 pixels) was used to mimic, as accurately as possible, natural interaction with a virtual environment. A single computer controlled the eye-tracker and collected all the data. Gaze positions were obtained at $120 \mathrm{~Hz}$ with an average precision of 0.5 degrees of visual angle $(5$ to $6 \mathrm{~mm}$ on the screen depending on the distance between the eye-tracker screen and the participants). Eye movements were analyzed using Tobii Studio 2.0.6 software. Eye fixations were defined as any period where the gaze remained focused for $58 \mathrm{~ms}$ (seven successive gaze points) or more within an area of 30 pixels in diameter (about $10 \mathrm{~mm}$ or $0.9 \mathrm{deg}$ of visual angle).

\section{Material}

The visual search material was created using Adobe Director 11 software. Two backgrounds were designed based on a pattern used by Tozzi, Morrone, and Burr (2007), namely a vertical, white (RGB: $255,255,255)$ to grey (RGB: $179,179,179)$ sinusoidal grating of 0.17 cycle/deg $(0.17$ cycle/cm). The "lateral" background only displayed vertical stripes (Figure 1, left and middle panels). The "radial" background displayed a radial visual structure with similar stripes of increasing length oriented in four different directions between the centre and the four sides of the screen (Figure 1, right panel). The stripes were horizontal at the top and bottom of the screen and vertical at the sides.

In Experiment 1a, three distinct versions of the backgrounds were used (Figure 1), namely the stationary and moving versions of the lateral background, and the moving version of the radial background. In the laterally moving background, the stripes drifted leftwards at a speed of $121 \mathrm{~mm} / \mathrm{s}$ (about $11 \mathrm{deg} / \mathrm{s}$ ). In the radially moving background, the stripes drifted at $121 \mathrm{~mm} / \mathrm{s}$ in each direction to simulate forward motion in a corridor.

Ten geometrical shapes of $12 \mathrm{~mm}$ in size were used as targets and distractors for the visual search task. These were squares, circles, triangles, hexagons and diamonds drawn in black and 
were either filled or empty. The search display always consisted of nine shapes arranged in a $170 \mathrm{~mm}$ (about $15 \mathrm{deg}$ ) diameter virtual circle (Figure 1). The shapes were arranged in the circle at a distance of $61 \mathrm{~mm}$ (about $5.5 \mathrm{deg}$ ) from one another.

[Figure 1 near here]

\section{Design and procedure}

The participants had to push a button to indicate whether one of the geometrical shapes was present or absent among the nine shapes presented on the eye-tracker screen. The nature of the background and the presence / absence of the target were manipulated at withinparticipant level. At the beginning of each block of trials, a target shape was shown at the centre of the screen for 5 seconds. Next, one of the backgrounds was displayed for 30 seconds to ensure full $\mathrm{OKN}$ activation. An empty blue square measuring $74 \mathrm{~mm}$ per side (about 6.7 deg) was then superimposed at the centre of the screen for 3 seconds to orient the participant's gaze towards the centre of the display without suppressing the OKN. The participants were instructed to focus their gaze as far as possible within the square. The square then disappeared and the background was displayed alone for 1 second. The search display appeared and the participants had to answer as quickly and accurately as possible by pushing one of two buttons labelled "absent" or "present".

Thirty blocks of four trials each were presented to each participant, with a short pause between each block. The target was present in half of the trials. All the trials in each block were performed against the same background and with the same target shape. When a moving background was used, the motion was continuous for the whole block. Target position within the search display was randomized. If the target was an empty (or filled) shape, the search displays contained only empty (or filled) shapes. When present, the target (e.g. a square) was 
presented between two exemplars of each of the other shapes (e.g. diamonds, circles, triangles and hexagons). When the target was absent, one of the other shapes was repeated three times. The order of presentation of the different backgrounds and the ten possible target shapes was randomized. However, each of the shapes was used as the target for one block of trials against each background.

\section{Dependent measures}

Search performance was assessed using response times (in milliseconds) as the dependent variable. Response times were measured between the appearance of the shapes and the participant's answer. Three dependent variables were obtained from participants' eye movements: the mean duration of eye fixations, and the horizontal (X) and vertical (Y) coordinates of the location of the initial fixation registered on the eye-tracker screen when the search display appeared. The location of the initial fixation was used to evaluate, in isolation, the impact of each background on the participants' average gaze position (Caroux, Le Bigot, and Vibert 2010; Ilg 1997). All the variables were analyzed using repeated measures ANOVAs with the nature of the background and the presence / absence of the target as within-participants factors. When the Mauchley sphericity test was significant, a GreenhouseGeisser correction was applied.

\section{Results}

All incorrect, premature (less than $100 \mathrm{~ms}$ ) and late (more than $3000 \mathrm{~ms}$ ) responses were excluded from the analysis. The proportion of excluded trials ranged from $0.8 \%$ to $13.3 \%$ across participants $(M=5.8 \%)$ and did not vary significantly across conditions. Response time data were logarithmically transformed before ANOVAs were performed. 


\section{Response time}

As shown in Table 1, the nature of the background had an impact on response times $(F(2,54)$ $\left.=8.80, p<.001, \eta_{p}^{2}=.25\right)$. Planned comparisons demonstrated that response times were longer for the radially moving background than for the stationary $(F(1,27)=8.72, p<.01)$ and laterally moving $(F(1,27)=15.77, p<.001)$ backgrounds. The presence / absence of the target also had an effect on response times $\left(F(1,27)=447.13, p<.001, \eta_{p}^{2}=.94\right)$, which were longer when the target was absent $(M=1591 \mathrm{~ms}, S D=291)$ than when it was present $(M=1149 \mathrm{~ms}, S D=195)$. The interaction between the two factors did not reach significance $(F(2,54)=2.46, p=.09)$.

[Table 1 near here]

\section{Eye movements}

There was no significant effect of either the nature of the background $(F(2,54)<1)$ or the presence/absence of the target $(F(1,27)=3.58, p=.07)$ on fixation durations and there was also no significant interaction between the two factors $(F(2,54)=1.70, p=.19)$.

As shown in Figure 2, there was an effect of the nature of the background on the X coordinate of the initial fixation $\left(F(2,54)=51.68, p<.001, \eta_{p}^{2}=.66\right)$. Planned comparisons indicated that this was located further to the right on the laterally moving background ( $M=678$ pixels, $S D=43)$ than on the stationary $(M=620$ pixels, $S D=31, F(1,27)=72.02, p<.001)$, and radially moving $(M=626$ pixels, $S D=22, F(1,27)=58.26, p<.001)$ backgrounds. The nature of the background also had an impact on the $\mathrm{Y}$ coordinate of the initial fixation $(F(2$, 54) $\left.=31.41, p<.001, \eta^{2}=.54\right)$, which was located further down and closer to the screen centre on the radially moving background $(M=489$ pixels, $S D=49)$ than on the stationary $(M$ 
$=451$ pixels, $S D=62, F(1,27)=32.51, p<.001)$ and laterally moving $(M=445$ pixels, $S D=$ $61, F(1,27)=41.17, p<.001)$ backgrounds. No other comparison reached significance.

[Figure 2 near here]

\section{Discussion}

\section{Impact of background motion}

The main hypothesis was not substantiated. When the background was moving, visual search performance decreased only for the radial background. The absence of impact of lateral background motion contrasts with previous data from Kaminiarz, Krekelberg, and Bremmer (2007) and Tozzi, Morrone, and Burr (2007), who observed that lateral background motion decreased performance in target detection tasks. The present data are closer to those of Menozzi and Koga (2004), who failed to show a significant impact of lateral background motion on reading performance. This result therefore suggests that the level of attention to stationary visual items required by the visual search task is sufficient to prevent performance impairment due to lateral background motion.

The second hypothesis was confirmed. Compared to the stationary background, the participants' gaze was deviated towards the right against the laterally moving background and towards the centre of the display against the radially moving background. Consequently, the participants' gaze were always deviated towards the origin of the movement. This finding strongly suggests that the two moving backgrounds triggered an OKN before the search display appeared. However, the OKN triggered by lateral background motion apparently had no impact on visual search performance. This might be because the shapes were used to 
cancel out the OKN (Ilg 1997; Pola, Wyatt, and Lustgarten 1992; Rubinstein and Abel 2011; Wyatt and Pola 1984).

The impairment of visual search performance by the radially moving background suggests that radial background motion has a greater impact than lateral motion on visual searches. However, an alternative interpretation could be that the visual search was not impaired because of the radial motion per se, but because of the particular visual structure used to create this radial motion. Indeed, radial backgrounds are more complex than lateral backgrounds, and visually complex backgrounds have a stronger impact on visual tasks than simpler backgrounds (Jie and Clark 2008; Wolfe et al. 2002). As a result, visual searches may be impaired by the radially moving background because of its greater complexity rather than because of the background motion itself. However, if this is the case, one must assume that, in the present experiment, the greater visual complexity of the radial background was insufficient to induce the significant increase in fixation durations that should have been observed according to Henderson, Chanceaux, and Smith (2009). The increase in the duration of fixations may actually be masked by the OKN suppression mechanisms.

To determine why the visual search was impaired by radial background motion, Experiment $1 \mathrm{~b}$ was conducted in order to determine whether or not a visual search would be impaired in the same way in response to the stationary version of the radial background. Experiment $1 \mathrm{~b}$ therefore compared the impact of stationary and moving radial backgrounds on a visual search. The hypothesis was that performance is poorer with the moving than with the stationary radial background due to the OKN triggered by radial motion (Ilg 1997). 


\section{Experiment 1b}

\section{Methods}

Fifteen volunteers (13 women) aged $M=18.9$ years $(S D=1.3)$ took part in the experiment. All participants had normal or corrected-to-normal vision. Seven participants were regular players, while all the others were occasional players.

The apparatus and visual search task were the same as in Experiment 1a. The radially moving background used in Experiment 1a was compared with a stationary version of the same background (Figure 1).

The motion of the background and the presence / absence of the target were manipulated within-participants. Twenty blocks of four trials each were presented to each participant, and the procedure and dependent measures were otherwise identical to those used in Experiment 1a.

\section{Results}

Again, all incorrect, premature and late responses were excluded from the analysis. Data from two participants for whom the proportion of excluded trials exceeded $15 \%$ were not analysed. For the other 13 participants, the proportion of excluded trials ranged from $2.8 \%$ to $10.0 \%$ ( $M$ $=4.6 \%$ ) and did not vary significantly across conditions.

As shown in Table 1, background motion had no significant impact on participants' response times $(F(1,12)=1.90, p=.19)$. The presence / absence of the target had an impact on response times $\left(F(1,12)=102.8, p<.001, \eta_{p}^{2}=.90\right)$, which were longer when the target was absent $(M=1696 \mathrm{~ms}, S D=178$ versus $M=1236 \mathrm{~ms}, S D=143)$. There was no significant interaction between the two factors $(F(1,12)<1)$.

The presence / absence of the target had an impact on fixation durations $(F(1,12)=27.8, p<$ $\left..001, \eta_{p}^{2}=.70\right)$. Fixations were longer when the target was present $(M=163 \mathrm{~ms}, S D=33$ 
versus $M=150 \mathrm{~ms}, S D=28$ ). There was no effect of background motion on fixation durations $(F(1,12)<1)$ and no significant interaction between the two factors $(F(1,12)=$ $1.03, p=.33)$.

Finally, as shown in Figure 3, there was no significant effect of background motion on either the $\mathrm{X}(F(1,12)<1)$ or $\mathrm{Y}$ coordinate $(F(2,54)<1)$ of the initial fixation. No other comparison reached statistical significance.

[Figure 3 near here]

\section{Discussion}

Contrary to expectations, visual search performance was not significantly impaired by radial background motion. Consequently, the impact of the radially moving background observed in Experiment 1a was linked to the greater visual complexity of the radial compared to the lateral background and not to background motion per se. In addition, radial background motion had no significant effect on the position of the initial fixation. This suggests that, in the case of the radially moving background, the participants' gazes were attracted towards the salient, central point of the visual structure rather than being deviated towards the origin of the background motion.

The mean duration of fixations was longer when the target was present in Experiment 1b, and actually tended to be longer in Experiment 1a. This may be due to the fact that the final fixation on the target shape, which occurred while the participants were making their response, was generally much longer than the other fixations.

Overall, the data from Experiments 1a and 1b suggest that the main determinant of visual search performance was the visual complexity of the background rather than whether or not the background was moving. Visual performance is apparently not affected by background 
motion if the task involves enough stationary objects on which the observer has to maintain his or her attention. As in other complex tasks such as reading (Menozzi and Koga 2004), people performing visual searches must fixate the different items in the display and this would be sufficient to attenuate or suppress OKN and/or the sensation of vection (Ilg 1997; Pola, Wyatt, and Lustgarten 1992; Rubinstein and Abel 2011; Wyatt and Pola 1984), and cancel out any negative impact they may have on the task (Menozzi and Koga 2004; Seno, Ito, and Sunaga 2011). 


\section{Experiment 2}

The aim of Experiment 2 was to investigate the impact of background motion and visual complexity on players' performance in a less attention-demanding task in which participants had to shoot at single targets as quickly as possible. The four backgrounds used in Experiments 1a and $1 \mathrm{~b}$ were tested in a single experiment. To check whether the simple presence of a patterned background had an impact on performance, these backgrounds were compared with a plain, grey background.

Because, in comparison to the visual search task, the shooting task is closer to the target detection task used by Kaminiarz, Krekelberg, and Bremmer (2007) and Tozzi, Morrone, and Burr (2007), the participants should have been less able to suppress OKN than in Experiment 1a. Thus, the first hypothesis was that shooting performance is poorer when the background moves than when it is stationary, but only in the case of the lateral background which does not permit OKN suppression. In the case of the radial background, participants may use the fixed, salient lines that separate the elements of the background to suppress OKN depending on the side of the screen to which they move. Because the radial background is visually more complex than the lateral one, the second hypothesis was that, independently of background motion, performance is poorer on the radial than on the lateral background. Similarly, the third hypothesis was that performance is poorer when the game background contains a visual pattern (whether lateral or radial) than when a plain background is used. In line with Henderson, Chanceaux, and Smith (2009), greater background complexity should also be associated with longer average fixation durations. 


\section{Methods}

\section{Participants}

Thirty-eight volunteers ( 24 women) aged $M=19.9$ years $(S D=2.8)$ took part in the experiment. All participants had normal or corrected-to-normal vision. Seventeen participants were regular players, thirteen were former regular players and eight were occasional players.

\section{Apparatus and material}

The apparatus was the same as in Experiments 1a and 1b. The material was created with Adobe Director 11 software. Five different backgrounds were used (Figure 4), namely the stationary and moving versions of the lateral and radial backgrounds and a plain grey (RGB: $217,217,217)$ control background. The target in the shooting task was a green creature $85 \mathrm{x}$ 66 pixels in size ( $22 \times 17 \mathrm{~mm}$, about 2.0 deg of visual angle). The cursor appeared as a weapon sight 114 pixels in diameter $(30 \mathrm{~mm})$.

[Figure 4 near here]

\section{Design and procedure}

The visual structure of the background (lateral or radial) was manipulated between participants. Participants were randomly assigned to one of the two conditions subject to the proviso that there was no significant difference in video game expertise between groups. In both cases, the background could be the stationary or moving version of the structured background or the plain grey background. This latter factor (i.e. the nature of the background) was manipulated within participants.

The procedure was presented as a video game. The participants were instructed to play the same video game several times and to aim to get a high score by playing as fast as possible. 
At the beginning of each trial, one of the backgrounds was displayed, and the empty blue square used in the previous experiments was shown for 1 second to orient the participants' gaze towards the centre of the display. The target and cursor then appeared at a random horizontal position on the screen. The cursor was located on the central, vertical axis of the screen, whereas the target was displayed at a random distance to the right or left. The participants had to aim for the target by moving the cursor horizontally with the left and right arrow keys, and to shoot at it with the "space" key. The participants could move the cursor and shoot as often as they wished until the target was hit, i.e. as long as the cursor and target were not superimposed when the shot was fired. When the target was hit, another trial began. In each game, the participants were instructed to hit as many enemies as possible in 60 seconds. At the end of each game, the participants were given feedback in the form of a score indicating their performance.

Each participant played twenty-four games, with a pause between each game. All trials for a game were performed with the same background. The order of presentation of the 24 games (8 games x 3 different backgrounds) was randomized.

\section{Dependent measures}

The participants' performances were assessed using the average time needed to hit the target (shooting time) as dependent measure. As in previous experiments, participants' eye movements were analysed based on the mean fixation duration and the location of the initial fixation on the eye-tracker screen when the target appeared. All variables were analyzed using repeated measures ANOVAs, with the structure of the background as a between-participants factor, and the nature of the background as within-participants factor. Shooting time data were logarithmically transformed before ANOVAs were performed. 


\section{Results}

\section{Shooting time}

As shown in Figure 5, the nature of the background had an impact on shooting times $(F(2,72)$ $=26.85, p<.001, \eta_{p}^{2}=.43$ ). There was no significant effect of background structure (lateral or radial, $F(1,36)<1)$, but there was a significant interaction between the two factors $(F(2,72)$ $\left.=15.29, p<.001, \eta_{p}^{2}=.30\right)$. Planned comparisons showed that with the radial structure, shooting times were longer for the stationary $(F(1,36)=39.36, p<.001)$ and moving $(F(1$, $36)=79.56, p<.001)$ versions of the radial background than for the plain background. In the case of the lateral structure, shooting times were longer for the laterally moving than for the stationary lateral background $(F(1,36)=9.11, p<.01)$ or the plain background $(F(1,36)=$ $7.74, p<.01)$

[Figure 5 near here]

\section{Duration of fixations}

The structure of the background had an impact on fixation durations $(F(1,36)=4.16, p<.05$, $\left.\eta_{p}^{2}=.10\right)$. Fixations were longer for the radial $(M=189 \mathrm{~ms}, S D=46)$ than for the lateral background $(M=162 \mathrm{~ms}, S D=40)$. The nature of the background also had an impact on fixation durations $\left(F(2,72)=4.05, p<.05, \eta_{p}^{2}=.10\right)$. Fixations were longer for the stationary $(M=179 \mathrm{~ms}, S D=48)$ and moving $(M=178 \mathrm{~ms}, S D=48)$ versions of the patterned backgrounds $(F(1,36)=5.62, p<.05$ and $F(1,36)=7.17, p<.05$, respectively) than for the plain background $(M=171 \mathrm{~ms}, S D=40)$. The interaction between the structure and nature of the background was not significant $(F(2,72)=1.02, p=.37)$. 


\section{Location of the initial fixation}

There was no effect of the structure $(F(1,36)<1)$ or nature $(F(2,72)=2.51, p=.09)$ of the background on the $\mathrm{X}$ coordinate of the initial fixation, nor any significant interaction between these factors.

In contrast, Figure 6 shows that the nature of the background had an impact on the $Y$ coordinate of the initial fixation $\left(F(2,72)=10.64, p<.001, \eta_{p}^{2}=.23\right)$ which interacted with the structure of the background $\left(F(2,72)=14.39, p<.001, \eta^{2}{ }_{p}=.29\right)$. In the case of the radial structure, the initial fixation was located lower down on the stationary $(M=486$ pixels, $S D=$ $34)$ and moving $(M=494$ pixels, $S D=30)$ versions of the radial background $(F(1,36)=$ $56.79, p<.001$ and $F(1,36)=40.69, p<.001$, respectively) than on the plain background $(M$ $=464$ pixels, $S D=33)$. In the case of the lateral structure, the Y coordinate of the initial fixation did not differ significantly between the stationary $(M=476$ pixels, $S D=54)$ and moving $(M=467$ pixels, $S D=39)$ versions of the lateral background and the plain background $(M=471$ pixels, $S D=49$, all $F s(1,36)<2.48, p>.12)$.

[Figure 6 near here]

\section{Discussion}

As expected, background motion had an impact on performance only when the lateral background was used. In line with previous data (Kaminiarz, Krekelberg, and Bremmer 2007; Tozzi, Morrone, and Burr 2007), lateral background motion had a detrimental impact on the simple shooting task used in Experiment 2, whereas it had no impact on visual search (Experiments 1a and 1b) or on reading performance (Menozzi and Koga 2004). This confirms that lateral background motion only has an impact on performance when the task is simple and does not involve sustained attention to stationary visual items. In contrast, and in the same 
way as in Experiment 1b, performance was not significantly affected by the motion of the radial background. Despite the impact of lateral background motion on performance, the deviation of participants' gaze towards the right side of the screen usually associated with OKN (Ilg 1997) was only present as a trend (Figure 6). In contrast, and as found in Experiment 1b, the participants' gaze was deviated towards the centre of the screen with both the stationary and moving versions of the radial background.

The second hypothesis was not fully substantiated. Although shooting times tended to be higher with the radial than with the lateral background, the difference was not significant. However, in line with Henderson, Chanceaux, and Smith (2009), fixations were longer for the more complex radial background than for the lateral background independently of background motion.

The third hypothesis was borne out in the case of the radial background only. Shooting performance was lower with the stationary radial background than the plain background. In contrast, the stationary lateral background did not impair shooting performance compared to the plain background. Nevertheless, eye fixations were longer for both the stationary and moving versions of the lateral and radial background than for the plain background. In other words, fixations were longer when the scene background displayed a visual pattern rather than no pattern. This finding is again consistent with Henderson, Chanceaux, and Smith (2009). Overall, the data confirm that the duration of fixations during a task is positively related to the visual complexity of the scene background, whether or not this background is moving. Indeed, fixations were longer with the lateral than with the plain background, and with the more complex radial than with the lateral background. However, contrary to previous suggestions (Jie and Clark 2008; Wolfe et al. 2002), background complexity does not always significantly impair task performance. One explanation may be that the attention paid to the superimposed visual elements used in the task attenuates the impact of background 
complexity. Alternatively, the difference in visual complexity between the radial and lateral backgrounds may not be sufficiently large to reveal complexity-related effects.

In sum, these findings demonstrate that in a simple shooting task, background motion and visual complexity combine to influence players' performance. High visual complexity seems to be more disruptive than background motion. When visually complex backgrounds are used, performance is impaired compared to a plain background, whether or not the background is moving. With the less complex lateral background, performance is impaired compared to a plain background only if the background is moving. 


\section{Conclusions and outlook}

In three experiments, background motion and visual complexity were manipulated together while participants performed either a visual search task or a classic shooting task. Both background motion and visual complexity had an impact on performance in the shooting task. In contrast, performance in the visual search task, which involved more sustained attention to superimposed visual items that may be used to cancel out $\mathrm{OKN}$, depended only on the visual complexity of the background. Further experiments must be performed to determine whether these principles hold true for other types of background motion encountered in video games such as angular motion (yaw, roll or pitch), and for combinations of different types of background motion (e.g. simulation of simultaneous rightward and forward motion). One limit of the present study is that OKN (i.e. the presence of alternative slow and phase phases of eye movement) could not be directly observed because of the low sampling rate of the eye tracker. The presence of OKN was only assessed indirectly by measuring the shift of observers' average gaze orientations on the screen. Hence, the present study does not unambiguously demonstrate that $\mathrm{OKN}$ is causing the performance impairments and eye movement modifications observed on moving backgrounds. According to So, Ho, and Lo (2001), the combination of background motion and visual complexity, which can be quantified by a "spatial velocity" metric, can induce cybersickness. Since the presence of cybersickness can impair performance in virtual tasks (Golding and Kerguelen 1992; Muth, Walker, and Fiorello 2006), part of the impact of moving backgrounds observed in the present study might be due to cybersickness.

Eye movement recordings demonstrated that whatever the task, the low-level features of the visual scene had a strong influence on gaze behaviour and fixation durations, in many cases explaining why the visual complexity or motion of the background impacted task performance. In fact, fixation durations were a more sensitive index of background 
complexity than task performance itself. This suggests that objectively measured physiological events such as eye movements may tell us more about the mechanisms involved in human performance than the typical operational measurements used in ergonomics. For instance, Jennett et al. (2008) demonstrated that the feeling of being deeply immersed in a game was associated with players who fixated the game display less often but for longer periods. Further experiments will be needed in order to gain a better understanding of how fixation durations are related to the local and global visual complexity of dynamic visual displays, and how this complexity relates to feelings of immersion in a game.

\section{Practical implications}

The present data may have practical implications for the design of virtual environments and video games. The radial backgrounds that are increasingly being used in virtual environments to simulate self-motion in three-dimensional settings (for instance in "first-person" views) will probably make it more difficult to interact with fixed, superimposed visual objects or contextual information. Designers should therefore be aware that introducing visual depth and the third dimension has a cost in terms of users' performance. In this situation, however, background motion would not have any great additional impact on performance. When a less complex background, such as a lateral background, is used, background motion will have less impact on users' performance provided that the task that is to be performed demands sustained attention to superimposed visual items. Nevertheless, lateral background motion should be avoided when the task is simpler and does not give participants the possibility to suppress OKN.

The above recommendations must be adapted for video game design. Indeed, while the purpose of ergonomic recommendations is generally to facilitate human-system interactions in the context of productive tasks (Nielsen 1993), game designers must create optimized challenges that will ensure that players remain motivated (Johnson and Wiles 2003; Malone 
and Lepper 1987; Pagulayan et al. 2012). Generally, challenges are created by increasing game difficulty which is linked to the amount of cognitive resources needed to complete each task (e.g., Qin, Rau, and Salvendy 2010).

The present data suggest that different low-level features of the scene background can be manipulated to modify the difficulty of a game, as has already been demonstrated by Jie and Clark (2008) in the case of background visual complexity. Interactions between the visual complexity and motion of the background should be taken into account when determining the difficulty of a game task. When the main task is simple and does not involve sustained attention to stationary items, game designers should not use visually complex or moving backgrounds if they want to keep game difficulty as low as possible. When the main task is more attention-demanding, visually complex background structures should also be avoided. In this case, however, background motion should not increase game difficulty. To increase game difficulty, game designers have to reverse these recommendations, keeping in mind that displaying a moving and visually complex background will not increase game difficulty further when the task is simple. 


\section{Acknowledgments}

Loïc Caroux was supported by a Ph.D. fellowship from the Direction Générale de l'Armement (DGA, French Ministry of Defence) and followed in the frame of this fellowship by Didier Bazalgette. 


\section{References}

Asher, M. F., D. J. Tolhurst, T. Troscianko, and I. D. Gilchrist. 2013. "Regional Effects of Clutter on Human Target Detection Performance." Journal of Vision 13 (5): article 25, 1-15. doi:10.1167/13.5.25.

Bae, S., H. Lee, H. Park, H. Cho, J. Park, and J. Kim. 2012. “The Effects of Egocentric and Allocentric Representations on Presence and Perceived Realism: Tested in Stereoscopic 3D Games.” Interacting with Computers 24: 251-264. doi:10.1016/j.intcom.2012.04.009.

Bos, J. E., W. D. Ledegang, A. J. A. Lubeck, and J. F. Stins. in press. "Cinerama Sickness and Postural Instability.” Ergonomics. doi:10.1080/00140139.2013.817614.

Bubka, A., and F. Bonato. 2010. "Natural Visual-field Features Enhance Vection.” Perception 39: 627-635. doi:10.1068/p6315.

Caroux, L., L. Le Bigot, and N. Vibert. 2010. "Searching for an Item in Dynamic Visual Environments: Impact of a Moving Background on Visual Search.” Perception 39 (ECVP Abstract Supplement): 192. doi:10.1068/v100088.

- 2011. "Maximizing Players' Anticipation by Applying the Proximity-compatibility Principle to the Design of Video Games." Human Factors 53: 103-117. doi:10.1177/0018720811400600.

Castel, A. D., J. Pratt, and E. Drummond. 2005. "The Effects of Action Video Game Experience on the Time Course of Inhibition of Return and the Efficiency of Visual Search.” Acta Psychologica 119: 217-230. doi:10.1016/j.actpsy.2005.02.004.

Chang, C.-H., W.-W. Pan, L.-Y. Tseng, and T. A. Stoffregen. 2012. "Postural Activity and Motion Sickness During Video Game Play in Children and Adults." Experimental Brain Research 217: 299-309. doi:10.1007/s00221-011-2993-4.

Chen, Y.-C., X. Dong, F.-C. Chen, and T. A. Stoffregen. 2012. "Control of a Virtual Avatar Influences Postural Activity and Motion Sickness.” Ecological Psychology 24: 279299. doi:10.1080/10407413.2012.726181.

Dyson, B. J. 2010. “'She's a Waterfall': Motion Aftereffect and Perceptual Design in Video Games Involving Virtual Musicianship.” Perception 39: 131-132. doi:10.1068/p6607.

Eckstein, M. P. 2011. "Visual Search: A Retrospective.” Journal of Vision 11 (5): article 14, 1-36. doi:10.1167/11.5.14.

Frey, A., J. Hartig, A. Ketzel, A. Zinkernagel, and H. Moosbrugger. 2007. "The Use of Virtual Environments Based on a Modification of the Computer Game Quake III 
Arena (R) in Psychological Experimenting." Computers in Human Behavior 23: 2026-2039. doi:10.1016/j.chb.2006.02.010.

Golding, J. F., and M. Kerguelen. 1992. "A Comparison of the Nauseogenic Potential of Lowfrequency Vertical Versus Horizontal Linear Oscillation.” Aviation, Space, and Environmental Medicine 63: 491-497.

Harrison, W. J., M. B. Thompson, and P. M. Sanderson. 2010. "Multisensory Integration with a Head-mounted Display: Background Visual Motion and Sound Motion.” Human Factors 52: 78-91. doi:10.1177/0018720810367790.

Henderson, J. M., M. Chanceaux, and T. J. Smith. 2009. "The Influence of Clutter on Realworld Scene Search: Evidence from Search Efficiency and Eye Movements.” Journal of Vision 9 (1): article 32, 1-8. doi:10.1167/9.1.32.

Hou, J., Y. Nam, W. Peng, and K. M. Lee. 2012. "Effects of Screen Size, Viewing Angle, and Players' Immersion Tendencies on Game Experience.” Computers in Human Behavior 28: 617-623. doi:10.1016/j.chb.2011.11.007.

Hubert-Wallander, B., C. Green, M. Sugarman, and D. Bavelier. 2011. "Changes in Search Rate but Not in the Dynamics of Exogenous Attention in Action Videogame Players." Attention, Perception, \& Psychophysics 73: 2399-2412. doi:10.3758/s13414-0110194-7.

Huestegge, L., and R. Radach. 2012. "Visual and Memory Search in Complex Environments: Determinants of Eye Movements and Search Performance.” Ergonomics 55: 10091027. doi:10.1080/00140139.2012.689372.

Hutchinson, C. V., and R. Stocks. 2013. "Selectively Enhanced Motion Perception in Core Video Gamers." Perception 42: 675-677. doi:10.1068/p7411.

Ilg, U. J. 1997. “Slow Eye Movements.” Progress in Neurobiology 53: 293-329. doi:10.1016/S0301-0082(97)00039-7.

Jennett, C., A. L. Cox, P. Cairns, S. Dhoparee, A. Epps, T. Tijs, and A. Walton. 2008. "Measuring and Defining the Experience of Immersion in Games." International Journal of Human-Computer Studies 66: 641-661. doi:10.1016/j.ijhcs.2008.04.004.

Ji, J. T. T., R. H. Y. So, and R. T. F. Cheung. 2009. "Isolating the Effects of Vection and Optokinetic Nystagmus on Optokinetic Rotation-Induced Motion Sickness." Human Factors 51: 739-751. doi:10.1177/0018720809349708. 
Jie, L., and J. J. Clark. 2008. "Video Game Design Using an Eye-movement-dependent Model of Visual Attention." ACM Transactions on Multimedia Computing Communications and Applications 4 (3): article 22, 1-16. doi:10.1145/1386109.1386115.

Johnson, D., and J. Wiles. 2003. "Effective Affective User Interface Design in Games." Ergonomics 46: 1332-1345. doi:10.1080/00140130310001610865.

Kaminiarz, A., B. Krekelberg, and F. Bremmer. 2007. "Localization of Visual Targets During Optokinetic Eye Movements.” Vision Research 47: 869-878. doi:10.1016/j.visres.2006.10.015.

Kim, J., and S. Palmisano. 2010. "Eccentric Gaze Dynamics Enhance Vection in Depth." Journal of Vision 10 (12): article 7, 1-11. doi:10.1167/10.12.7.

Lepecq, J.-C., C. De Waele, S. Mertz-Josse, C. Teyssèdre, P. T. B. Huy, P.-M. Baudonnière, and P.-P. Vidal. 2006. "Galvanic Vestibular Stimulation Modifies Vection Paths in Healthy Subjects.” Journal of Neurophysiology 95: 3199-3207. doi:10.1152/jn.00478.2005.

Malone, T. W., and M. R. Lepper. 1987. "Making Learning Fun: A Taxonomy of Intrinsic Motivations for Learning." In Aptitude, Learning, and Instruction: III. Conative and Affective Process Analysis, edited by R. E. Snow and M. J. Farr, 223-253. Hillsdale, NJ: Lawrence Erlbaum.

Menozzi, M., and K. Koga. 2004. "Visual Information Processing in Augmented Reality: Some Aspects of Background Motion.” Swiss Journal of Psychology 63: 183-190. doi:10.1024/1421-0185.63.3.183.

Merhi, O., E. Faugloire, M. Flanagan, and T. A. Stoffregen. 2007. "Motion Sickness, Console Video Games, and Head-mounted Displays.” Human Factors 49: 920-934. doi:10.1518/001872007x230262.

Muth, E. R., A. D. Walker, and M. Fiorello. 2006. "Effects of Uncoupled Motion on Performance.” Human Factors 48: 600-607. doi:10.1518/001872006778606750.

Nielsen, J. 1993. Usability Engineering. San Francisco: Morgan Kaufmann.

Pagulayan, R., K. Keeker, T. Fuller, D. Wixon, R. Romero, and D. Gunn. 2012. "UserCentered Design in Games." In Human-Computer Interaction Handbook, edited by J. Jacko, 795-822. Boca-Raton, FL: CRC Press.

Pola, J., H. Wyatt, and M. Lustgarten. 1992. "Suppression of Optokinesis by a Stabilized Target: Effects of Instruction and Stimulus Frequency." Perception \& Psychophysics 52: 186-200. doi:10.3758/bf03206772. 
Pölönen, M., T. Järvenpää, and B. Bilcu. 2013. "Stereoscopic 3D Entertainment and Its Effect on Viewing Comfort: Comparison of Children and Adults." Applied Ergonomics 44: 151-160. doi:10.1016/j.apergo.2012.06.006.

Qin, H., P. L. P. Rau, and G. Salvendy. 2010. "Effects of Different Scenarios of Game Difficulty on Player Immersion.” Interacting with Computers 22: 230-239. doi:10.1016/j.intcom.2009.12.004.

Raj, R., W. S. Geisler, R. A. Frazor, and A. C. Bovik. 2005. "Contrast Statistics for Foveated Visual Systems: Fixation Selection by Minimizing Contrast Entropy." Journal of the Optical Society of America A: Optics Image Science and Vision 22: 2039-2049. doi:10.1364/JOSAA.22.002039.

Rayner, K. 2009. "Eye Movements and Attention in Reading, Scene Perception, and Visual Search.” Quarterly Journal of Experimental Psychology 62: 1457-1506. doi:10.1080/17470210902816461.

Riecke, B. E., J. Schulte-Pelkum, M. N. Avraamides, M. Von Der Heyde, and H. H. Bülthoff. 2006. "Cognitive Factors Can Influence Self-motion Perception (vection) in Virtual Reality." ACM Transactions on Applied Perception 3: 194-216. doi:10.1145/1166087.1166091.

Rubinstein, N. J., and L. A. Abel. 2011. "Optokinetic Nystagmus Suppression as an Index of the Allocation of Visual Attention." Investigative Ophthalmology \& Visual Science 52: 462-467. doi:10.1167/iovs.10-6016.

Sabri, A. J., R. G. Ball, A. Fabian, S. Bhatia, and C. North. 2007. "High-resolution Gaming: Interfaces, Notifications, and the User Experience.” Interacting with Computers 19: 151-166. doi:10.1016/j.intcom.2006.08.002.

Seno, T., H. Ito, and S. Sunaga. 2011. “Attentional Load Inhibits Vection.” Attention, Perception, \& Psychophysics 73: 1467-1476. doi:10.3758/s13414-011-0129-3.

So, R. H. Y., A. Ho, and W. T. Lo. 2001. “A Metric to Quantify Virtual Scene Movement for the Study of Cybersickness: Definition, Implementation, and Verification." Presence: Teleoperators and Virtual Environments 10: 193-215. doi:10.1162/105474601750216803.

Stanney, K. M., M. Mollaghasemi, L. Reeves, R. Breaux, and D. A. Graeber. 2003. "Usability Engineering of Virtual Environments (VEs): Identifying Multiple Criteria That Drive Effective VE System Design.” International Journal of Human-Computer Studies 58: 447-481. doi:10.1016/s1071-5819(03)00015-6. 
Stanney, K. M., R. R. Mourant, and R. S. Kennedy. 1998. "Human Factors Issues in Virtual Environments: A Review of the Literature." Presence: Teleoperators and Virtual Environments 7: 327-351. doi:10.1162/105474698565767.

Stoffregen, T. A., E. Faugloire, K. Yoshida, M. B. Flanagan, and O. Merhi. 2008. "Motion Sickness and Postural Sway in Console Video Games.” Human Factors 50: 322-331. doi:10.1518/001872008x250755.

Thornton, T. L., and D. L. Gilden. 2007. "Parallel and Serial Processes in Visual Search." Psychological Review 114: 71-103. doi:10.1037/0033-295x.114.1.71.

Tozzi, A., M. C. Morrone, and D. C. Burr. 2007. "The Effect of Optokinetic Nystagmus on the Perceived Position of Briefly Flashed Targets." Vision Research 47: 861-868. doi:10.1016/j.visres.2006.10.022.

Trutoiu, L. C., B. J. Mohler, J. Schulte-Pelkum, and H. H. Bülthoff. 2009. “Circular, Linear, and Curvilinear Vection in a Large-screen Virtual Environment with Floor Projection.” Computers \& Graphics 33: 47-58. doi:10.1016/j.cag.2008.11.008.

Vansteenkiste, P., G. Cardon, E. D’Hondt, R. Philippaerts, and M. Lenoir. 2013. “The Visual Control of Bicycle Steering: The Effects of Speed and Path Width." Accident Analysis \& Prevention 51: 222-227. doi:10.1016/j.aap.2012.11.025.

Waespe, W., and U. Schwarz. 1987. "Slow Eye Movements Induced by Apparent Target Motion in Monkey.” Experimental Brain Research 67: 433-435. doi:10.1007/bf00248564.

Wolfe, J. M. 1998. "What Can 1 Million Trials Tell Us About Visual Search?” Psychological Science 9: 33-39. doi:10.1111/1467-9280.00006.

Wolfe, J. M., A. Oliva, T. S. Horowitz, S. J. Butcher, and A. Bompas. 2002. "Segmentation of Objects from Backgrounds in Visual Search Tasks." Vision Research 42: 2985-3004. doi:10.1016/S0042-6989(02)00388-7.

Wolfson, S., and G. Case. 2000. "The Effects of Sound and Colour on Responses to a Computer Game.” Interacting with Computers 13: 183-192. doi:10.1016/S09535438(00)00037-0.

Wu, S., and I. Spence. 2013. "Playing Shooter and Driving Videogames Improves Top-down Guidance in Visual Search.” Attention, Perception, \& Psychophysics 75: 673-686. doi:10.3758/s13414-013-0440-2.

Wyatt, H. J., and J. Pola. 1984. “A Mechanism for Suppression of Optokinesis.” Vision Research 24: 1931-1945. doi:10.1016/0042-6989(84)90027-0. 
Yannakakis, G., H. Martínez, and A. Jhala. 2010. "Towards Affective Camera Control in Games." User Modeling and User-Adapted Interaction 20: 313-340. doi:10.1007/s11257-010-9078-0. 


\begin{tabular}{lcccc}
\hline Background & Stationary lateral & Laterally moving & Stationary radial & Radially moving \\
\hline Experiment 1a & $1361(348)$ & $1336(337)$ & - & $1413(311)$ \\
Experiment 1b & - & - & $1445(276)$ & $1486(291)$ \\
\hline
\end{tabular}

Table 1. Mean response times (in ms) obtained in Experiments 1a and $1 \mathrm{~b}$ according to the nature of the background. Standard deviations are shown in parentheses. 


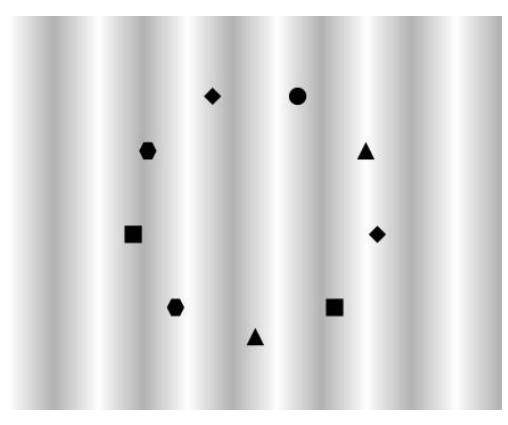

Stationary lateral

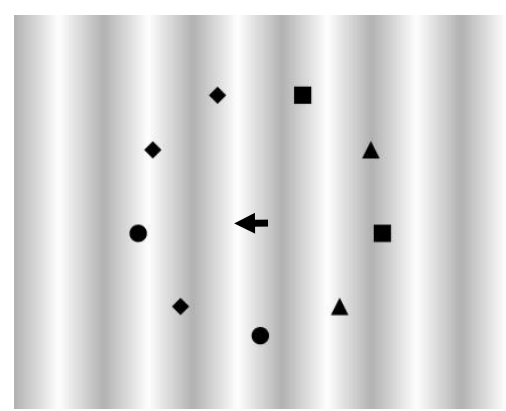

Laterally moving background

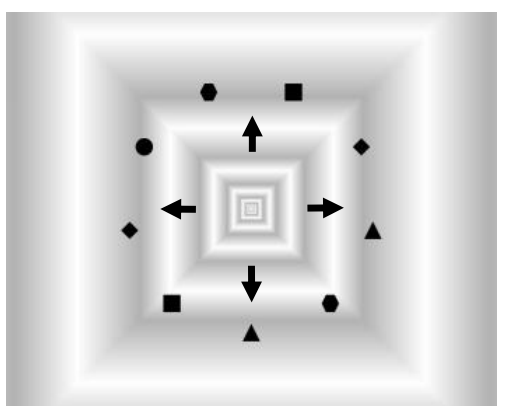

Radially moving background

background

Figure 1. Backgrounds used in Experiment 1a with examples of superimposed search displays. A stationary version of the radial background was added in Experiment $1 \mathrm{~b}$. The black arrows indicate the directions of background motion but were not shown on the displays. 


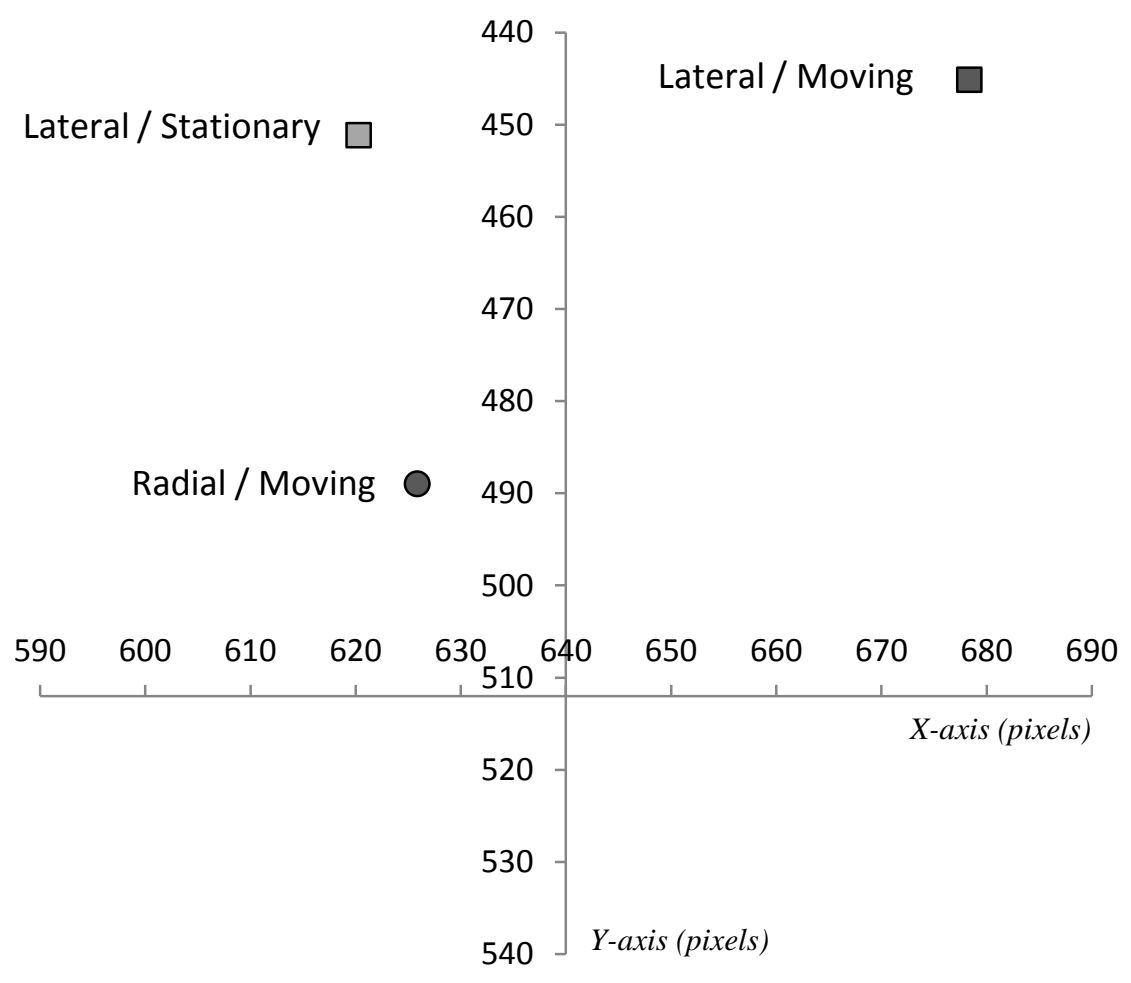

Figure 2. Close-up view of the central region of the eye-tracker screen showing the average coordinates (in pixels) of the initial fixation on the search display according to the nature of the background in Experiment 1a. The origin of the axes is at the centre of the screen. 


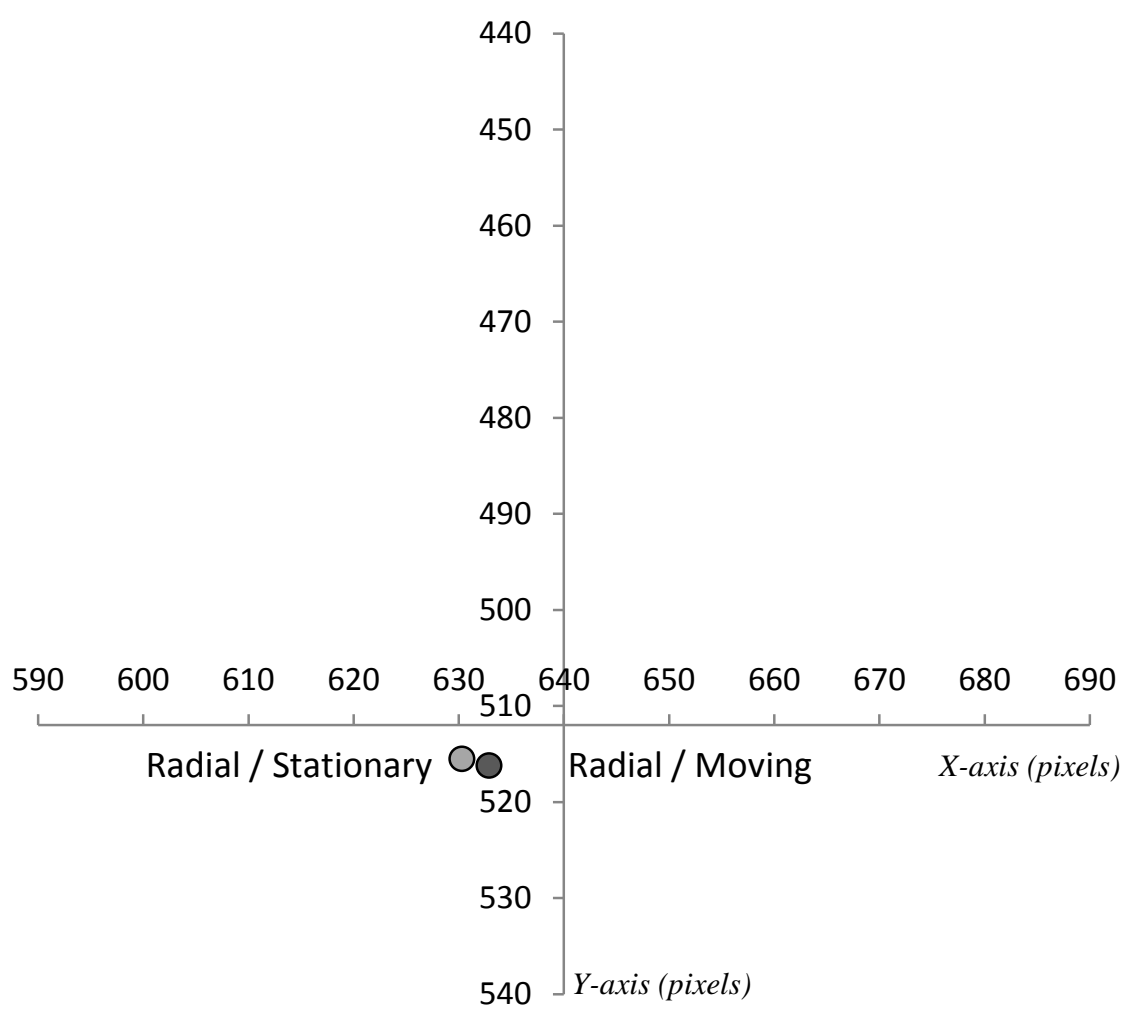

Figure 3. Close-up view of the central region of the eye-tracker screen showing the average coordinates (in pixels) of the initial fixation on the search display according to the nature of the background in Experiment 1b. The origin of the axes is the centre of the screen. 


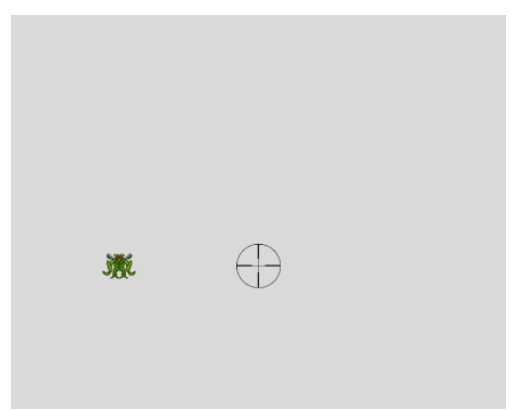

Plain background

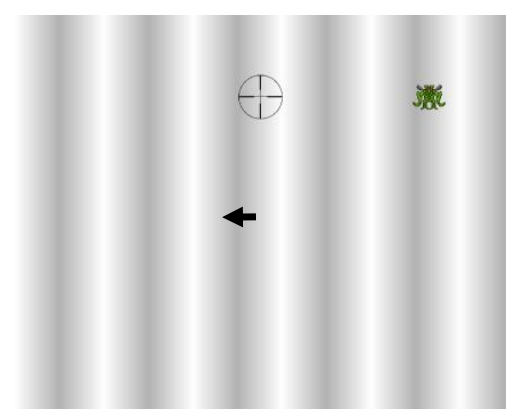

Lateral background

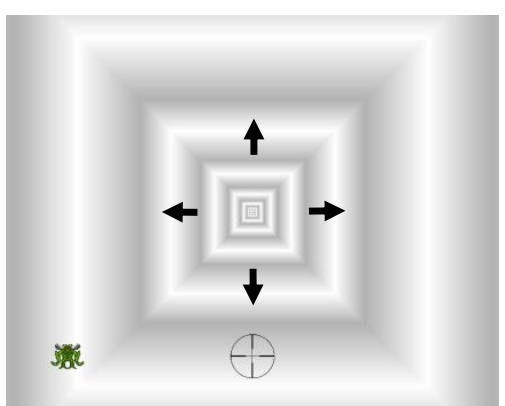

Radial background

Figure 4. Backgrounds used in Experiment 2 with examples of initial locations of the target and cursor. Both the stationary and moving versions of the lateral and radial backgrounds were used. The black arrows indicate the directions of background motion but were not shown on the actual displays. 


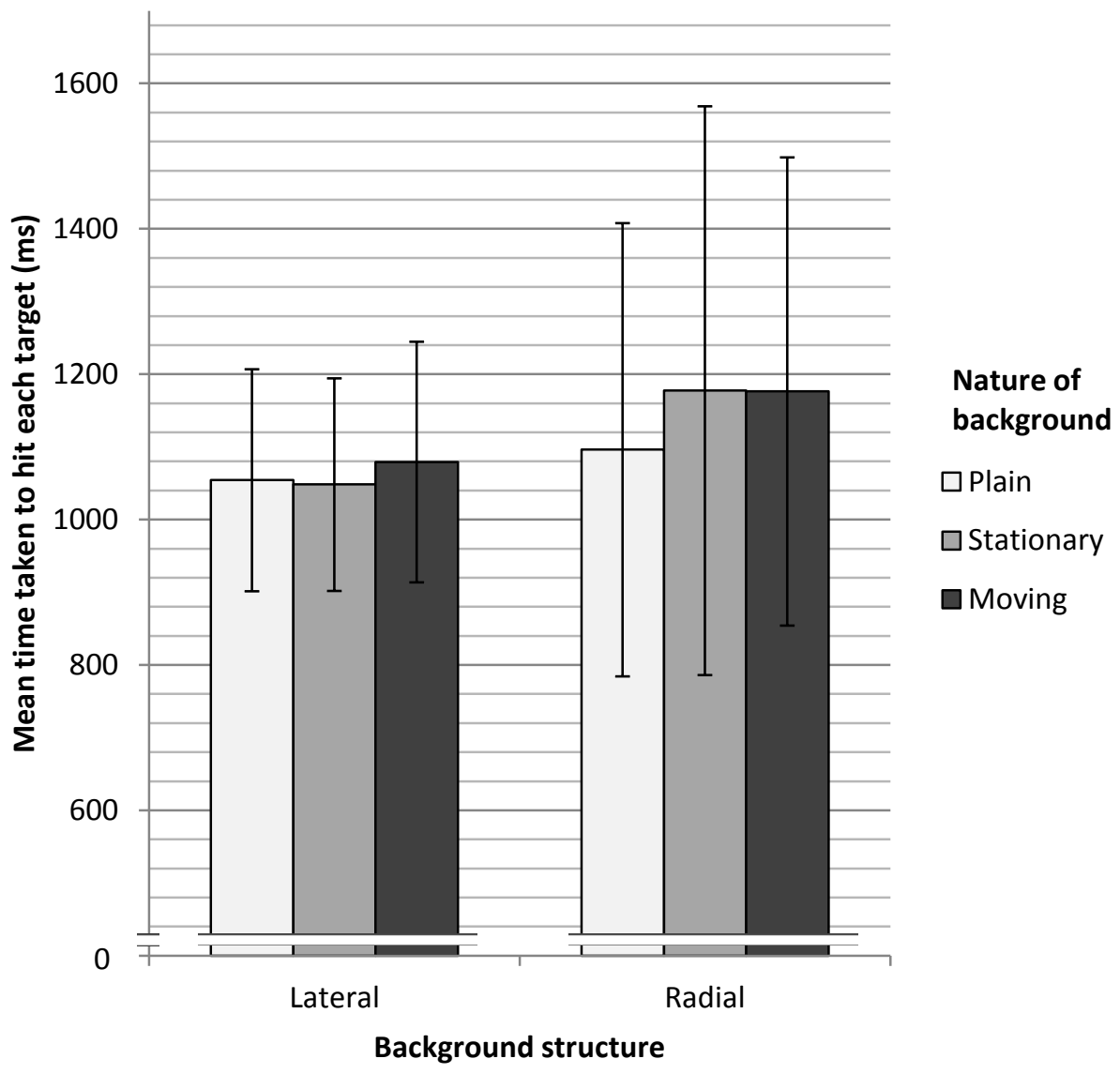

Figure 5. Mean time (in ms) taken to hit targets in the different experimental conditions used in Experiment 2. The error bars represent standard errors. 


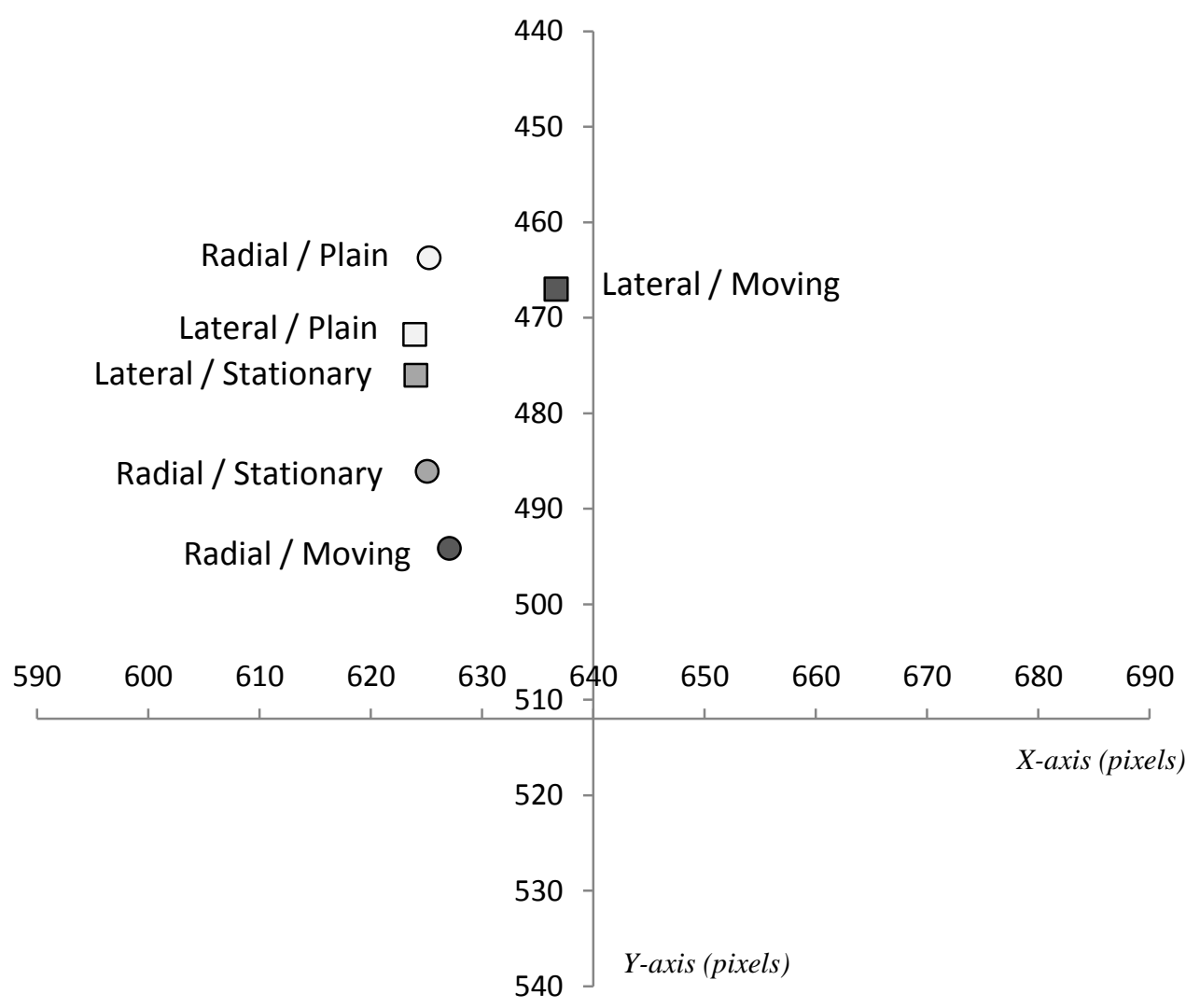

Figure 6. Close-up view of the central region of the eye-tracker screen showing the average coordinates (in pixels) of the initial fixation on the game display according to the nature and visual structure of the background in Experiment 2. The origin of the axes is the centre of the screen. 